\title{
SPMSM Sliding Mode Control Based on the New Super Twisting Algorithm
}

\author{
Lei Zhang $\mathbb{D}$, Jing Bai $\mathbb{D}$, and Jing Wu \\ College of Electrical and Information Engineering, Beihua University, Jilin City 132013, China \\ Correspondence should be addressed to Jing Bai; jlbyj@163.com
}

Received 8 June 2021; Revised 4 July 2021; Accepted 21 July 2021; Published 31 July 2021

Academic Editor: Weixiang Zhou

Copyright (C) 2021 Lei Zhang et al. This is an open access article distributed under the Creative Commons Attribution License, which permits unrestricted use, distribution, and reproduction in any medium, provided the original work is properly cited.

To achieve the high-performance control of the surface-mounted permanent magnet synchronous motor (SPMSM) speed control system, this paper proposes a high-order sliding mode control strategy based on a new super twisting algorithm (NSTA). This strategy introduces an adaptive term in its proportional term based on the original super twisting algorithm, which solves low reaching speed and poor antidisturbance ability due to the square root calculation of proportional term in the original super twisting algorithm. The simulation results show that the proposed strategy can effectively improve the system's response speed and antidisturbance and greatly suppress the chattering phenomenon of traditional sliding mode control.

\section{Introduction}

Surface-mounted permanent magnet synchronous motors are widely used in aerospace, numerical control systems, wind power generation, and new energy electric vehicle drive systems due to their high efficiency and small size $[1,2]$. However, it is a nonlinear, strong coupling, multivariable complex object. Although a traditional PI controller can meet the control requirements within a certain range, it cannot meet the requirements of high-performance control when system parameters change or are affected by external uncertainties.

To solve the problems caused by traditional PI control, scholars from all over the world have conducted much research, and some modern control theory achievements have been successfully applied to the speed control system of SPMSM, such as adaptive control, fuzzy control, neural network, active disturbance rejection control, and sliding mode control. Among them, sliding mode control is widely used because of its robustness, fast dynamic response, and easy implementation [3]. Although the traditional sliding mode control improves the robustness of the system, when the sliding mode control is applied to the actual system, due to the time delay and space lag of the switch, the error of the state detection, and other factors, it is easy to cause the system chattering phenomenon and reduce the dynamic quality of the system [4]. Therefore, how to suppress chattering is the key to the application of sliding mode control.

Chinese scholar Gao proposed a sliding mode control strategy based on the reaching law in 1996, which effectively improved the system's dynamic quality and reduced the sliding mode chattering to a certain extent [5]. The authors in literature [6] proposed a sliding mode control strategy based on exponential reaching law and Sigmoid function, which effectively suppressed sliding mode chattering. However, due to the introduction of the Sigmoid function, the convergence speed and stability of the system are reduced to a certain extent, and the exponential reaching law has contradictory problems between sliding mode chattering and reaching speed and robustness. The authors in literature $[7,8]$ designed a sliding mode control strategy based on integral sliding mode surface, which introduces the integration of state variables into the conventional sliding mode surface, which can effectively eliminate the steady-state error of speed and torque and accelerate the system response speed and at the same time it has strong robustness to load disturbance. However, the introduction of integral sliding mode surface is easy to produce integral saturation, which leads to large overshoot, which affects the quality of system 
control. The authors in literature [9-12] proposed a terminal sliding mode control strategy that improves the convergence of the system so that the system state converges to a given trajectory within finite time, but there is a singularity problem. Therefore, nonsingular terminal sliding mode control is proposed. It directly avoids controlling the singular area from the sliding mode design and retains the finite-time convergence characteristics of the terminal sliding mode. However, when the system state is far from the equilibrium point, its convergence time is relatively long and the dynamic characteristics worsen [13-15]. The author in literature [16] proposed a second-order sliding mode control: a super twisting algorithm composed of an integral and proportional term. The proportional term plays a role in reaching speed. When the moving point reaches the sliding mode surface, the integral term makes the system trajectory move around the origin to ensure the continuity of the output control signal and thus reduce the chattering phenomenon in the sliding mode control. In literature [17], the super twisting algorithm was applied to the SPMSM speed regulation system and compared with the sliding mode control based on exponential reaching law. It was found that the algorithm effectively solved the problem of the contradiction between chattering, reaching speed, and antidisturbance ability in the traditional sliding mode control under the premise of suppressing sliding mode chattering. However, the determination of the gain of this algorithm requires that the disturbance term is differentiable and bounded. In practical applications, this bound is difficult to determine. In order to ensure the convergence of the system, an excessively large gain value is often selected, which may cause serious system chattering-even crash. Therefore, the authors in literature [18] proposed a PMSM speed control system based on the adaptive super spiral algorithm, which effectively solved the problem of overestimating the gain of the existing algorithm and improved the system's stability. However, none of the above strategies consider insufficient reaching speed and poor antidisturbance ability of the super twisting algorithm because its proportional term is the square root calculation $[19,20]$.

In order to solve the above problems, this paper proposes a sliding mode control strategy based on a new super twisting algorithm. This strategy introduces an adaptive term in its proportional term based on the original super twisting algorithm, which solves low reaching speed and poor antidisturbance ability due to the square root calculation of proportional term in the original super twisting algorithm. On the premise of ensuring that the system chattering is not increased, the dynamic quality of the SPMSM speed control system is improved.

\section{The Motor Motion Equation of SPMSM}

In this paper, the SPMSM is taken as the research object, and its motion equation can be expressed as follows [1]:

$$
\frac{\mathrm{d} \omega}{\mathrm{d} t}=\frac{3 p_{n} \psi_{f}}{2} i_{q}-\frac{\beta}{J} \omega-\frac{T_{L}}{J},
$$

where $p_{n}$ is the pole pairs; $\psi_{f}$ is the rotor flux linkage; $\omega$ is the speed; $\beta$ is the viscosity friction coefficient; $J$ is the moment of inertia; $T_{L}$ is the load torque; and $i_{q}$ is the $q$-axis current.

\section{Sliding Mode Speed Controller Design}

3.1. Sliding Mode Controller Based on Exponential Reaching Law. The linear sliding surface is as follows:

$$
s=c x_{1}+x_{2}
$$

where $\omega^{*}$ is the reference speed of the motor; $x_{1}=\omega^{*}-\omega$; and $x_{2}=\dot{x}_{1}=-\dot{\omega}$.

In recent years, the sliding mode algorithm based on the reaching law has been widely used in SPMSM speed controller design because it can guarantee the dynamic quality of the reaching motion.

The exponential reaching law is as follows [13]:

$$
\dot{s}=-\varepsilon \operatorname{sign}(s)-q s,
$$

where $s$ is the sliding mode variable and $\varepsilon$ and $q$ are the sliding mode control switching gain.

Derivation of equation (2) is obtained, and combining with equation (1), we obtain the following:

$$
\begin{aligned}
\dot{s} & =c \dot{x}_{1}+\dot{x}_{2}=c x_{2}+\dot{x}_{2} \\
& =-c\left(\frac{3 p_{n} \psi_{f}}{2 J} i_{q}-\frac{\beta}{J} \omega-\frac{T_{L}}{J}\right)-\left(\frac{3 p_{n} \psi_{f}}{2 J} i_{q}-\frac{\beta}{J} \dot{\omega}\right) \\
& =c x_{2}-\frac{3 p_{n} \psi_{f}}{2 J} i_{q}-\frac{\beta}{J} x_{2} \\
& =\left(c-\frac{\beta}{J}\right) x_{2}-\frac{3 p_{n} \psi_{f}}{2 J} \dot{i}_{q} .
\end{aligned}
$$

Combined with equations (3) and (4), we obtain the following:

$$
\left(c-\frac{\beta}{J}\right) x_{2}-\frac{3 p_{n} \psi_{f}}{2 J} \dot{i}_{q}=-\varepsilon \operatorname{sign}(s)-q s .
$$

Thus, the design control law can be obtained as follows:

$$
\dot{i}_{q}=\frac{2 J}{3 p_{n} \psi_{f}}\left(\left(c-\frac{\beta}{J}\right) x_{2}+\varepsilon \operatorname{sign}(s)+q s\right) .
$$

Thus, the reference current of the $q$ axis can be obtained as follows:

$$
i_{q}^{*}=\frac{2 J}{3 p_{n} \psi_{f}} \int_{0}^{t}\left(\left(c-\frac{\beta}{J}\right) x_{2}+\varepsilon \operatorname{sign}(s)+q s\right) \mathrm{d} \tau .
$$

As shown in equation (7), chattering will occur in the system due to a discontinuous term $\varepsilon \operatorname{sign}(s)$ in the reference current. Secondly, the reaching speed, chattering, and robustness of the system are all related to the value of $\varepsilon$ and $q$. The larger $\varepsilon$ and $q$ are, the stronger the robustness and the faster the reaching are, but the chattering will also be more 
significant. Therefore, there is a contradiction between system chattering and reaching speed and robustness.

The Lyapunov function is defined as follows:

$$
V=\frac{1}{2} s^{2} .
$$

The derivative of $V$ can be obtained as follows:

$$
\dot{V}=s \dot{s} .
$$

Combined with equations (1), (4), and (6), we can get

$$
\begin{aligned}
\dot{V} & =s \dot{s}=s\left(\left(c-\frac{B}{J}\right) x_{2}-\frac{3 p_{n} \psi_{f}}{2 J} i_{q}\right) \\
& =s(-\varepsilon \operatorname{sign}(s)-q s) \\
& =-\left(\varepsilon \operatorname{sign}(s) s+q s^{2}\right),
\end{aligned}
$$

where both are normal numbers and $\varepsilon \operatorname{sign}(s) s+q s^{2}$ is always greater than 0 , so $\dot{V}<0$. According to the Lyapunov stability criterion, the SMC speed controller based on the exponential reaching law is stable.

3.2. Speed Controller Design Based on NSTA-SMC. The highorder sliding mode algorithm provides a solution for the contradiction between system chattering and reaching speed. Unlike other high-order sliding mode algorithms, the super twisting algorithm does not need to derive the sliding mode surface so that the sliding mode surface and its derivative can be stabilized to zero simultaneously, avoiding that the introduction of noise control law design is simple. So, its general expression is as follows [14]:

$$
\begin{aligned}
u & =-\alpha|s|^{1 / 2} \operatorname{sign}(s)+u_{1}, \\
\dot{u}_{1} & =-\beta \operatorname{sign}(s)+\dot{\varphi},
\end{aligned}
$$

where $s$ is the sliding mode variable; $\varphi$ is the disturbance term; $\alpha$ and $\beta$ are sliding mode gain coefficients; and $\operatorname{sign}()$ is the switching function.

From equation (11), it can be seen that the proportional term $\alpha|s|^{1 / 2} \operatorname{sign}(s)$ plays a role in improving the reaching speed of the algorithm, but its sliding mode surface is calculated by square root, and the gain of the scale term directly affects the reaching speed and antidisturbance ability of the algorithm. In order to improve the antidisturbance ability and reaching speed of the algorithm, a new super twisting algorithm is proposed in this paper, namely:

$$
\begin{aligned}
u & =-\alpha|s|^{1 / 2} \operatorname{sign}(s)-k s+u_{1}, \\
\dot{u}_{1} & =-\beta \operatorname{sign}(s)+\dot{\varphi},
\end{aligned}
$$

where $k s$ is a linear term and $k>0$.

The sliding surface is selected as follows:

$$
s=x=\omega^{*}-\omega .
$$

Derivation of equation (13) gives

$$
\dot{s}=\dot{x}=-\dot{\omega} .
$$

Combined with equations (1) and (14), the output of the controller is as follows:

$$
\dot{s}=-\dot{\omega}=-\frac{3 p_{n} \psi_{f}}{2 J} i_{q}+\frac{B}{J} \omega+\frac{T_{L}}{J} .
$$

Combined with equations (12) and (15), the output of the controller is as follows:

$$
i_{q}^{*}=\frac{2 J}{3 p_{n} \psi_{f}}\left(\left(\frac{B}{J} \omega+\frac{T_{L}}{J}\right)+\alpha|s|^{1 / 2} \operatorname{sign}(s)+k s+\int \beta \operatorname{sign}(s) \mathrm{d} t\right) .
$$

\subsection{System Stability Analysis}

Lemma 1 (see [21]). For equation (11), if $|\dot{\varphi}| \leq \delta$ and $\delta>0$, then $\alpha$ and $\beta$ satisfy the following values:

$$
\left\{\begin{array}{l}
\alpha>2, \\
\beta>\frac{\alpha^{3}+(4 \alpha-8) \delta^{2}}{\alpha(4 \alpha-8)} .
\end{array}\right.
$$

Then, equation (11) can converge to the origin in finite time.

Theorem 1. For equation (16), if $2|\dot{\varphi}| \leq \delta$ and $\delta>0$, then $\alpha, \beta$, and $k$ satisfy the following values:

$$
\left\{\begin{array}{l}
\alpha>2, \\
\beta>\frac{\left(\alpha+k\left|z_{1}\right|^{1 / 2}\right)^{2}}{4\left(\alpha+k\left|z_{1}\right|^{1 / 2}\right)-8}+\frac{\delta^{2}}{4 \alpha}, \\
k>0 .
\end{array}\right.
$$
time.

Then, equation (16) can converge to the origin in finite Let be $\left((B / J) \omega+\left(T_{L} / J\right)\right)=\varphi$, use variable substitution:

$$
\left\{\begin{array}{l}
z_{1}=s \\
z_{2}=-\int \beta \operatorname{sign}\left(z_{1}\right) \mathrm{d} t+\varphi .
\end{array}\right.
$$

Thus, equation (19) can be rewritten as follows:

$$
\left\{\begin{array}{l}
\dot{z}_{1}=-\alpha|z 1|^{1 / 2} \operatorname{sign}\left(z_{1}\right)-k z_{1}+z_{2}, \\
\dot{z}_{2}=-\beta \operatorname{sign}\left(z_{1}\right)+\dot{\varphi} .
\end{array}\right.
$$

For equation (16), the quasi-quadratic Lyapunov function [22] is selected as follows:

$$
V\left(z_{1}, z_{2}\right)=\zeta^{T} \Pi \zeta
$$

where $\zeta^{T}=\left[\zeta_{1}, \zeta_{2}\right]=\left[\left|z_{1}\right|^{1 / 2} \operatorname{sign}\left(z_{1}\right), z_{2}\right] ; \Pi$ is a real symmetric positive definite matrix; take 


$$
\Pi=\left[\begin{array}{cc}
4 \beta+\left(\alpha+k\left|z_{1}\right|^{1 / 2}\right)^{2} & -\left(\alpha+k\left|z_{1}\right|^{1 / 2}\right) \\
-\left(\alpha+k\left|z_{1}\right|^{1 / 2}\right) & 2
\end{array}\right] .
$$

According to equation (19), $V\left(z_{1}, z_{2}\right)$ is a radially unbounded continuous positive definite function and $V\left(z_{1}, z_{2}\right)$ is differentiable everywhere except for the set $\left\{z_{1}=0\right\}$. The derivative of $V\left(z_{1}, z_{2}\right)$ along the system trajectory can be obtained as follows:

$$
\begin{aligned}
\dot{V}\left(z_{1}, z_{2}\right) & =\dot{\xi}^{T} \Pi \xi+\xi^{T} \Pi \dot{\xi} \\
& =\frac{1}{2} \frac{1}{\left|\xi_{1}\right|}\left[\xi^{T} A^{T} \Pi \xi+\xi^{T} \Pi A \xi+\rho B^{T} \Pi \xi+\rho \xi^{T} \Pi B\right],
\end{aligned}
$$

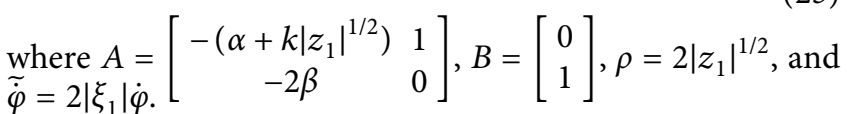

Obviously, $\rho=2\left|z_{1}\right|^{1 / 2}$ and $\tilde{\varphi}=2\left|\xi_{1}\right| \dot{\varphi}$ are scalars, and it can be seen from the expansion calculation of $B^{T} \Pi \xi$ and $\xi^{T} \Pi B$ that $B^{T} \Pi \xi$ and $\xi^{T} \Pi B$ are also scalars, and according to equation (22), $\Pi=\Pi^{T}$ can be seen, so

$$
B^{T} \Pi \xi=\left(B^{T} \Pi \xi\right)^{T}=\xi^{T} \Pi^{T}\left(B^{T}\right)^{T}=\xi^{T} \Pi B .
$$

Let be $m=B^{T} \Pi \xi=\xi^{T} \Pi B$ and $m^{2}=\xi^{T} \Pi B B^{T} \Pi \xi$ according to the inequality

$$
(m-\rho)^{2}=m^{2}-2 m \rho+\rho^{2} \geq 0, \quad \forall m, \rho \in R .
$$

From equation (25), we can know

$$
\xi^{T} \Pi B B^{T} \Pi \xi \geq \rho \xi^{T} \Pi B+\rho B^{T} \Pi \xi-\rho^{2} .
$$

According to Theorem 1, suppose the perturbation term $\varphi$ is Lipschitz continuous, and $2|\dot{\varphi}| \leq \delta, \delta>0$.

Combining $\rho=2\left|z_{1}\right|^{1 / 2}$ and $\dot{\varphi}=2\left|\xi_{1}\right| \dot{\varphi}$, we obtain

$$
\delta^{2} \xi_{1}^{2}-\rho^{2} \geq 0
$$

Let be $C=\left[\begin{array}{ll}1 & 0\end{array}\right]$, we obtain

$$
\delta^{2} \xi_{1}^{2}=\delta^{2} \xi^{T} C^{T} C \xi .
$$

Combining equations (23) to (28), it can be concluded that

$$
\begin{aligned}
\dot{V}\left(z_{1}, z_{2}\right) \leq & \frac{1}{2} \frac{1}{\left|\xi_{1}\right|}\left[\left(\xi^{T} A^{T} \Pi \xi+\xi^{T} \Pi A \xi+\rho B^{T} \Pi \xi+\rho \xi^{T} \Pi B\right)\right. \\
& \left.+\delta^{2} \xi_{1}^{2}-\rho^{2}\right] \\
\leq & \frac{1}{2} \frac{1}{\left|\xi_{1}\right|}\left[\xi^{T} A^{T} \Pi \xi+\xi^{T} \Pi A \xi+\delta^{2} \xi^{T} C^{T} C \xi\right. \\
& \left.+\xi^{T} \Pi B B^{T} \Pi \xi\right] \\
= & \frac{1}{2} \frac{1}{\left|\xi_{1}\right|} \xi^{T}\left[A^{T} \Pi+\Pi A+\delta^{2} C^{T} C+\Pi B B^{T} \Pi\right] \xi \\
= & -\frac{1}{2} \frac{1}{\left|\xi_{1}\right|} \xi^{T} Q \xi,
\end{aligned}
$$

where $Q=-\left[A^{T} \Pi+\Pi A+\delta^{2} C^{T} C+\Pi B B^{T} \Pi\right]$.

Obviously, for equation (29), when $Q$ is a positive definite matrix, we obtain

$$
\dot{V}\left(z_{1}, z_{2}\right)=-\frac{1}{2} \frac{1}{\left|\xi_{1}\right|} \xi^{T} Q \xi<0 .
$$

Thus, the Lyapunov function $V(z)$ of the system shown in equation (16) satisfies the stability conditions in the Lyapunov stability theory of $V>0$, which is radially unbounded and $\dot{V}<0$.

By further expanding and calculating $Q$, we can get

$$
\begin{aligned}
Q & =-\left[A^{T} \Pi+\Pi A+\delta^{2} C^{T} C+\Pi B B^{T} \Pi\right] \\
& =\left[\begin{array}{cc}
2\left(\alpha+k\left|z_{1}\right|^{1 / 2}\right)^{3}-\left(\alpha+k\left|z_{1}\right|^{1 / 2}\right)^{2}+4 \beta\left(\alpha+k\left|z_{1}\right|^{1 / 2}\right)-\delta^{2} & 2\left(\alpha+k\left|z_{1}\right|^{1 / 2}\right)-2\left(\alpha+k\left|z_{1}\right|^{1 / 2}\right)^{2} \\
2\left(\alpha+k\left|z_{1}\right|^{1 / 2}\right)-2\left(\alpha+k\left|z_{1}\right|^{1 / 2}\right)^{2} & 2\left(\alpha+k\left|z_{1}\right|^{1 / 2}\right)-4
\end{array}\right] .
\end{aligned}
$$

If $Q>0$, then $\dot{V}<0$; according to Schur's complement lemma [23], it can be deduced that a sufficient and necessary condition for $Q$ to be a positive definite matrix is

$$
\left\{\begin{array}{l}
\alpha+k\left|z_{1}\right|^{1 / 2}>2 \\
\beta>\frac{\left(\alpha+k\left|z_{1}\right|^{1 / 2}\right)^{3}+\delta^{2}\left(\alpha+k\left|z_{1}\right|^{1 / 2}\right)-2 \delta^{2}}{4\left(\alpha+k\left|z_{1}\right|^{1 / 2}\right)\left[\left(\alpha+k\left|z_{1}\right|^{1 / 2}\right)-2\right]} .
\end{array}\right.
$$

Since $k>0$, and in the existing system, there must be $\left|\omega^{*}-\omega\right|_{\max }^{1 / 2} \geq\left|z_{1}\right|^{1 / 2} \geq 0$, and equation (32) can be transformed into the following:

$$
\left\{\begin{array}{l}
\alpha>0, \\
\beta>\frac{\left(\alpha+k\left|z_{1}\right|^{1 / 2}\right)^{2}}{4\left(\alpha+k\left|z_{1}\right|^{1 / 2}\right)-8}+\frac{\delta^{2}}{4 \alpha} \\
k>0
\end{array}\right.
$$

The proof is completed. 


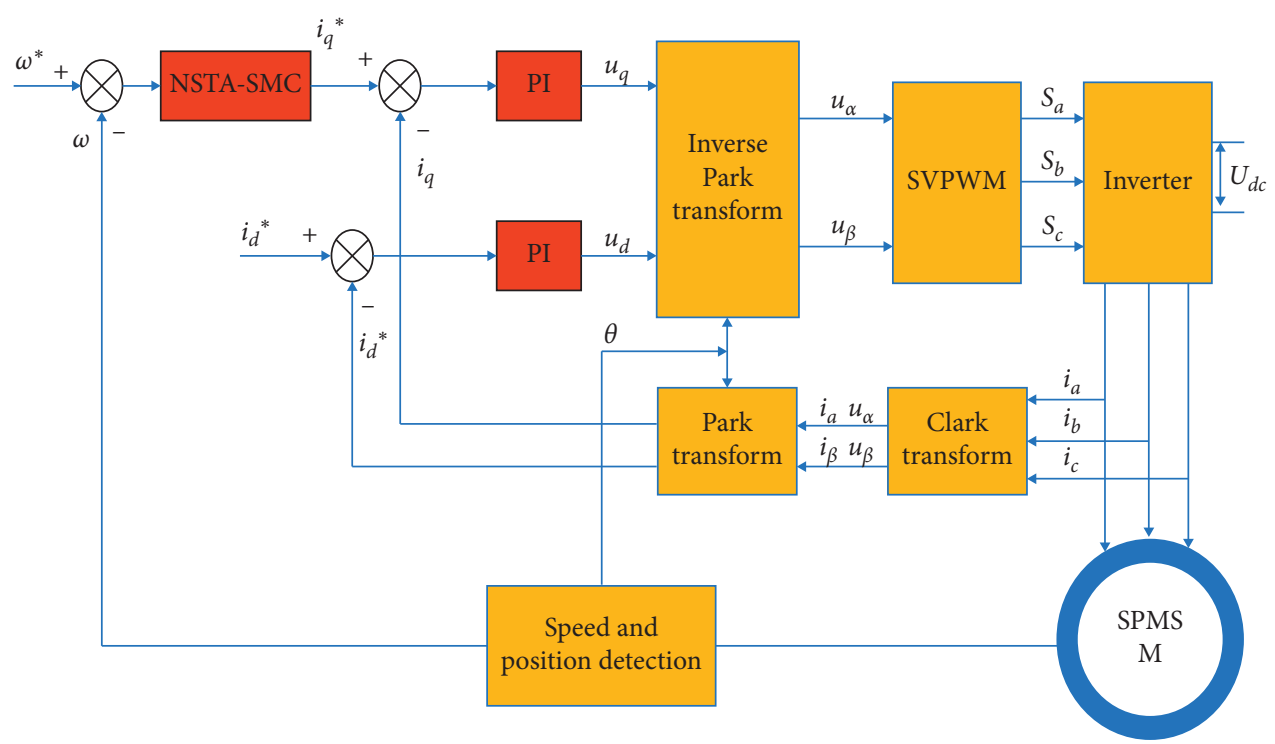

FIgURE 1: SPMSM speed regulation system based on NSTA-SMC.

TABLE 1: SPMSM parameters.

\begin{tabular}{lc}
\hline Parameters & Values \\
\hline Stator resistance $R s$ & $2.875 \Omega$ \\
Pole-pairs number & 4 \\
Magnetic flux $\psi_{f}$ & $0.175 \mathrm{~Wb}$ \\
$D$-axis induction $L d$ & $8.5 e-3 \mathrm{H}$ \\
$Q$-axis induction $L q$ & $8.5 e-3 \mathrm{H}$ \\
Damping factor $B$ & 0 \\
DC bus voltage $V$ & $311 \mathrm{~V}$ \\
Rotational inertia $J$ & $0.003 \mathrm{~kg} \cdot \mathrm{m}^{2}$ \\
\hline
\end{tabular}

3.4. The Influence of Linear Term on the Stability and Reaching Speed of the System. According to the above proof process, the linear term $k s$ is introduced into the proportional term of the super twisting algorithm, so long as $k>0$ is guaranteed, the system's stability will not be affected. At the same time, when the system state of the linear term $k s$ is close to the sliding mode surface, that is, $s<1, k s$ reaches 0 , the proportional term becomes the square root reaching law $\alpha|s|^{1 / 2} \operatorname{sign}(s)$, and there is still the problem of low reaching speed. In order to solve this problem, the adaptive term $k|s|^{b \cdot \operatorname{sign}(|s|-1)} s$ is introduced in this paper, and equation (16) becomes

$$
\begin{aligned}
i_{q}^{*}= & \frac{2 J}{3 p_{n} \psi_{f}}\left(\left(\frac{B}{J} \omega+\frac{T_{L}}{J}\right)+\alpha|s|^{1 / 2} \operatorname{sign}(s)+k|s|^{b \cdot \operatorname{sign}(|s|-1)} s\right. \\
& \left.+\int \beta \operatorname{sign}(s) \mathrm{d} t\right),
\end{aligned}
$$

where $k|s|^{b \cdot \operatorname{sign}(|s|-1)} s$ is the adaptive term, $k>0,0<b<1$.

When the system state is close to the sliding mode surface, i.e., $s<1$, then $\operatorname{sign}(|s|-1)=-1$ and the proportional term becomes $\alpha|s|^{1 / 2} \operatorname{sign}(s)+k|s|^{-b} s$, which is obviously $\alpha|s|^{1 / 2} \operatorname{sign}(s)+k|s|^{-b} s \gg \alpha|s|^{1 / 2} \operatorname{sign}(s)$ from the perspective of reaching speed. When the system state is far away from the sliding mode surface, i.e., $s>1$, then $\operatorname{sign}(|s|-$ $1)=1$ and the proportional term becomes $\alpha|s|^{1 / 2} \operatorname{sign}(s)+$ $k|s|^{b} s$, which is obviously $\alpha|s|^{1 / 2} \operatorname{sign}(s)+k|s|^{b} s \gg \alpha$ $|s|^{1 / 2} \operatorname{sign}(s)$ from the perspective of reaching speed.

\section{Simulation Research}

In order to verify the antidisturbance ability and chattering suppression ability of the strategy proposed in this paper, a simulation model was built in Matlab/Simulink according to Figure 1. SPMSM parameters used for simulation are shown in Table 1.

In order to prove the effectiveness of the proposed new super twisting algorithm, NSTA-SMC, STA-SMC, the sliding mode control based on the exponential reaching law (SMC), and PI control performance are compared and simulated. The premise is that the above four methods of current loop using PI controller and the parameters are the same. The simulation results are shown in Figure 2. Among them, NSTA-SMC parameters designed in this paper are $\alpha=1500, \beta=60000, k=600$, and $b=0.5$; PI parameter is $k_{p}=0.1$ and $k_{i}=3$; SMC1 parameter is $\varepsilon=500000, c=60$, and $q=300$; SMC2 parameter is $\varepsilon=800000, c=60$, and $q=300$; STA-SMC parameter is $\alpha=1500$ and $\beta=60000$.

Figure 2 shows the system adopts no-load start mode, and the given speed is $1000 \mathrm{rpm}$. From Figure 3 and Table 2, compared with PI, SMC1, SMC2, and STA-SMC, NSTASMC has the smallest starting overshoot, shortest regulation time, and fastest response speed. At $0.2 \mathrm{~s}$, the load changes to $10 \mathrm{~N} \cdot \mathrm{m}$. Figure 4 and Table 2 show that when the load changes suddenly, the PI speed drop is the largest and the regulation time is the longest. Compared with PI, the speed drop of SMC1 and SMC2 is smaller, and their size is closely related to the size of controller parameters $\varepsilon$ and $q$. Compared with STA-SMC, the speed drop of NSTA-SMC with the introduction of the adaptive term is $51.9 \%$ less than that of STA-SMC and it can quickly recover to a given speed, 


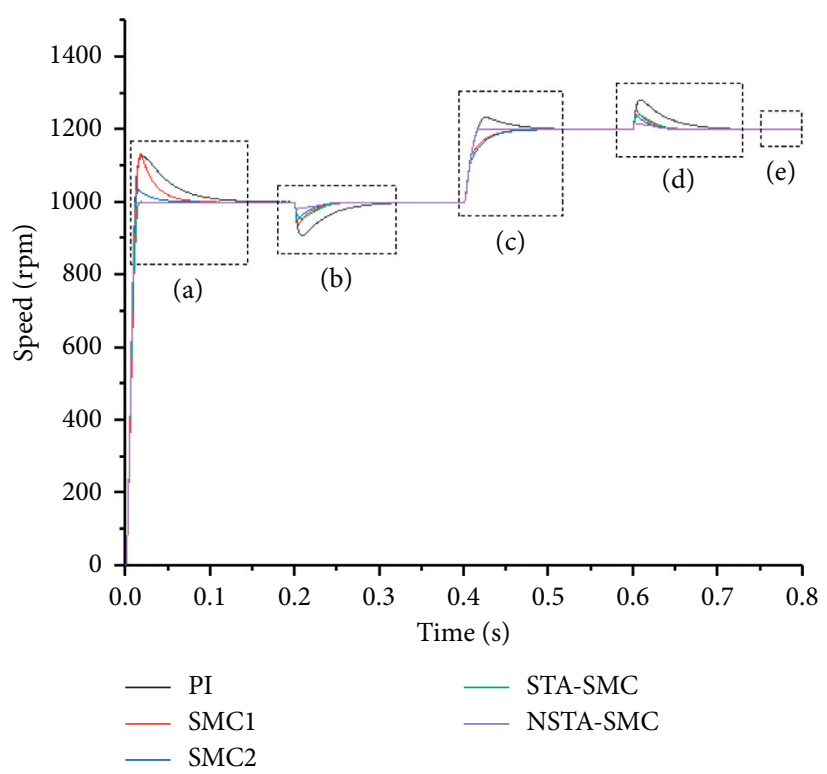

Figure 2: Speed response diagram.

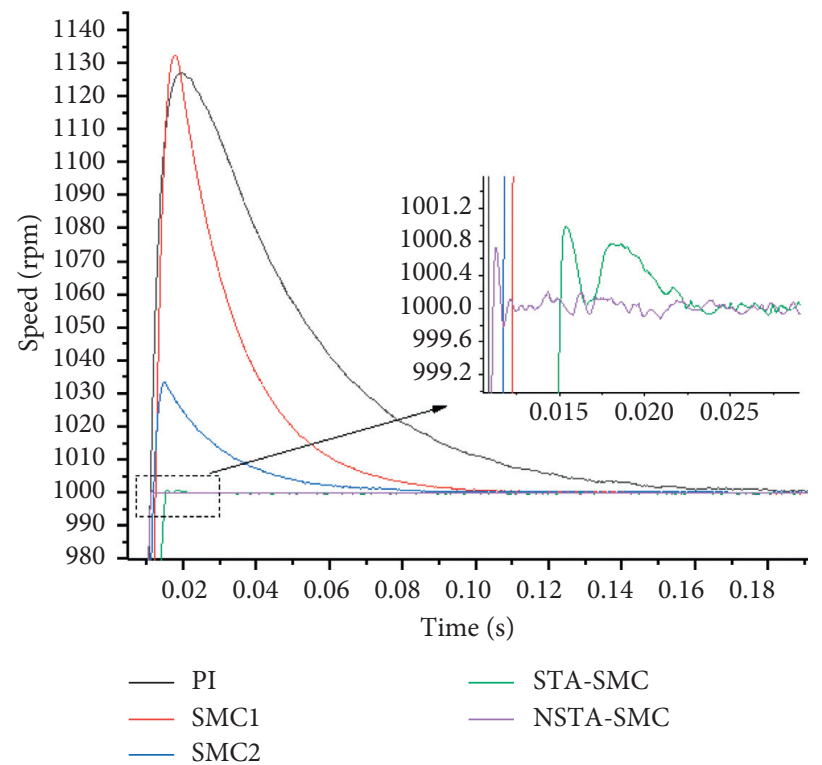

Figure 3: Enlargement of local speed (a).

TABLE 2: SPMSM system performance.

\begin{tabular}{|c|c|c|c|c|c|c|}
\hline Result & PI & SMC1 & SMC2 & STA-SMC & NSTA-SMC & Unit \\
\hline Response time & 0.18 & 0.1215 & 0.1215 & 0.0225 & 0.01175 & $s$ \\
\hline Regulation time 1 & 0.18 & 0.1 & 0.1 & 0.06 & 0.06 & s \\
\hline Regulation time 2 & 0.18 & 0.095 & 0.095 & 0.03 & 0.03 & s \\
\hline Regulation time 3 & 0.18 & 0.09 & 0.09 & 0.057 & 0.057 & s \\
\hline Start overshoot & 127.5 & 132.55 & 33.65 & 1 & 0.75 & $\mathrm{rpm}$ \\
\hline Load overshoot & 92 & 61.75 & 48.82 & 44.7 & 21.5 & $\mathrm{rpm}$ \\
\hline Speed change overshoot & 33.5 & 0 & 0 & 2.6 & 1.76 & $\mathrm{rpm}$ \\
\hline Unload overshoot & 82 & 52 & 40.6 & 39.4 & 17.4 & rpm \\
\hline Speed tracking error & 0.63 & 0.28 & 0.44 & 0.235 & 0.135 & $\mathrm{rpm}$ \\
\hline
\end{tabular}




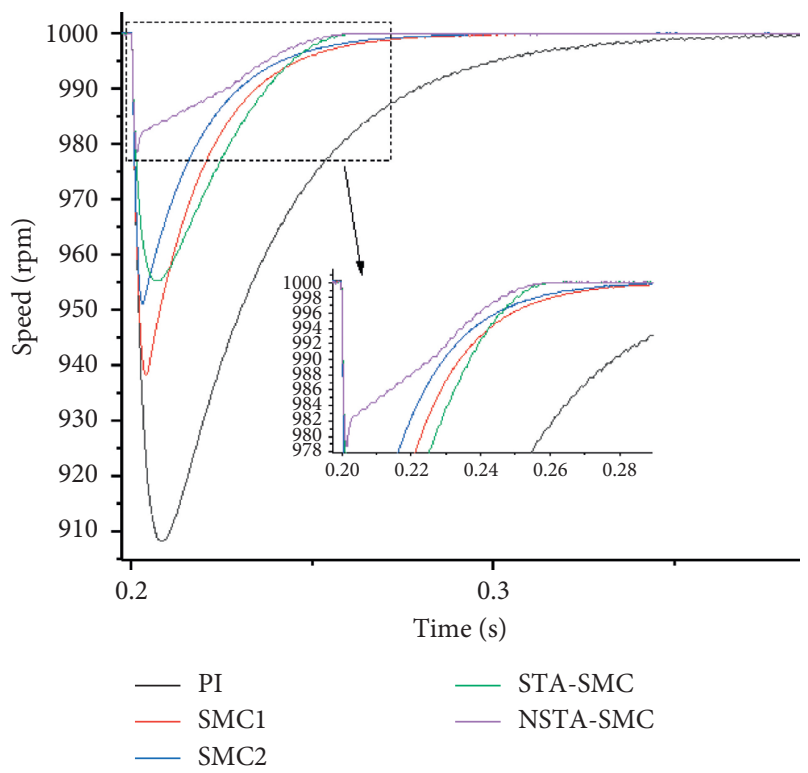

Figure 4: Enlargement of local speed (b).

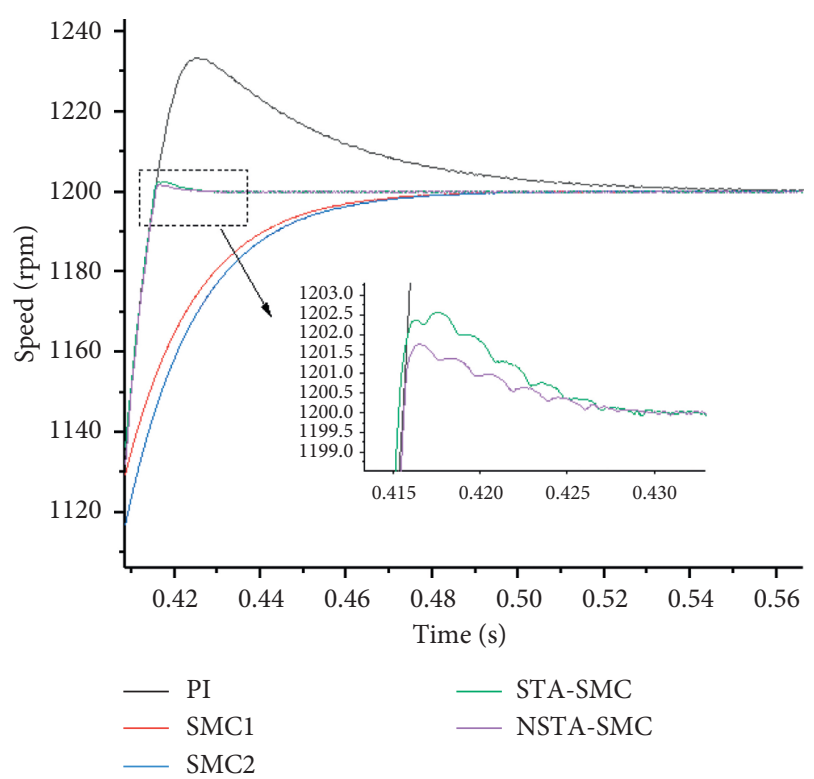

FIGURE 5: Enlargement of local speed (c).

which effectively solves the problem of low reaching speed of the original super twisting algorithm and further improves the antidisturbance ability of the system. At $0.4 \mathrm{~s}$, the speed suddenly rises to $1200 \mathrm{rpm}$. From Figure 5 and Table 2, when the speed changes suddenly, the PI overshoot is the largest and the regulation time is the longest; although SMC1 and SMC2 have no overshoot, the regulation time is much longer than that of STA-SMC and NSTA-SMC. At $0.6 \mathrm{~s}$, the load of $10 \mathrm{~N} \cdot \mathrm{m}$ is removed. From Figure 6 and Table 2, compared with PI, SMC1, SMC2, and STA-SMC, NSTA-SMC has the slightest change in speed and quickly recovers to a given speed, with good antidisturbance ability. From Figure 7 and Table 2, when the speed is $1200 \mathrm{rpm}$, the steady-state error of PI speed is the largest, and the control quality is poor. The

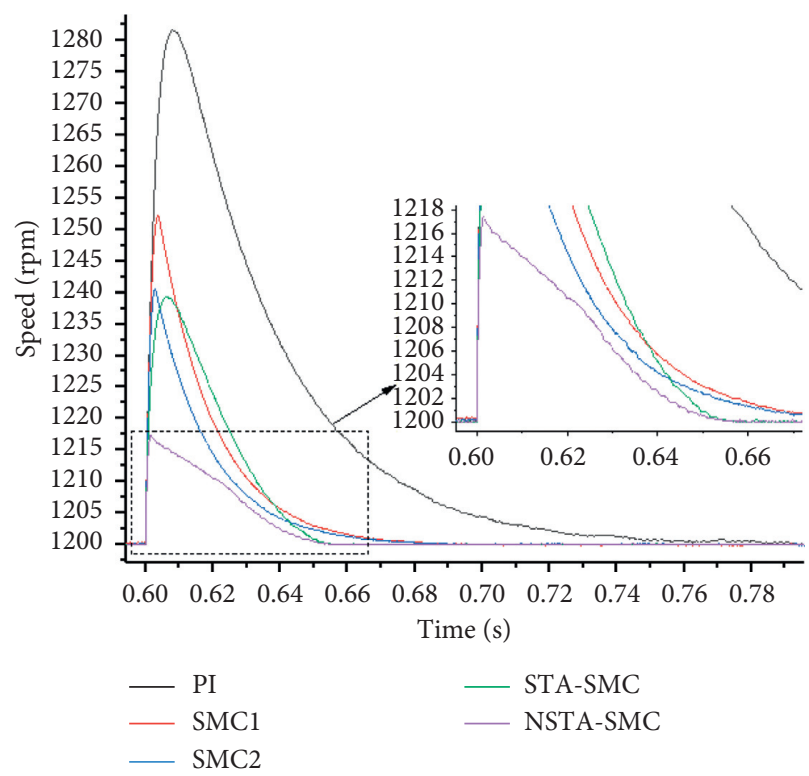

Figure 6: Enlargement of local speed (d).

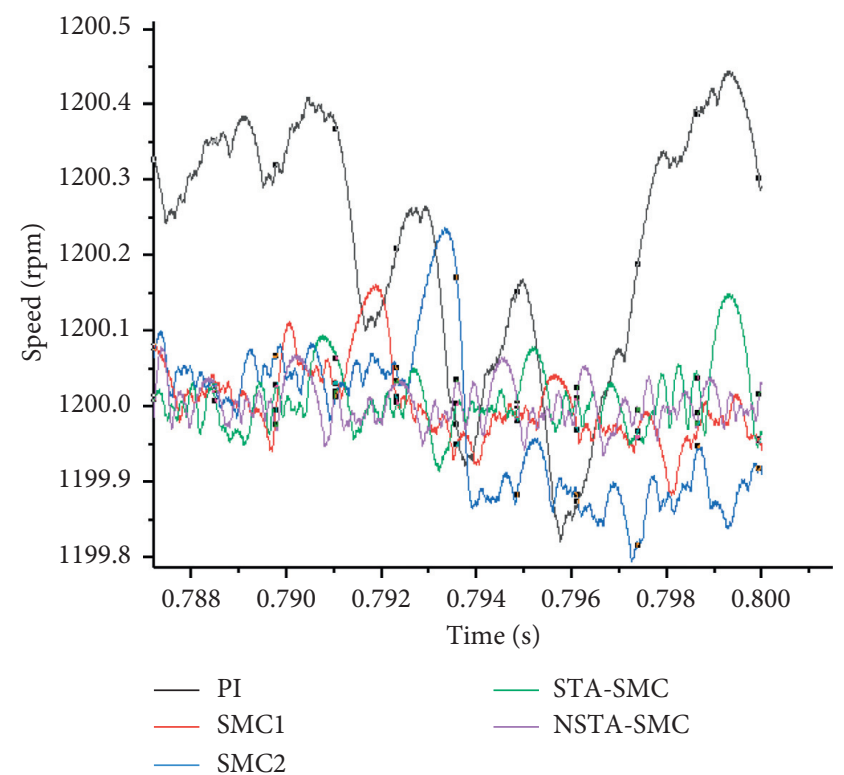

Figure 7: Enlargement of local speed (e).

system chattering of SMC1 and SMC2 is closely related to the size of the controller parameters $\varepsilon$ and $q$. Compared with SMC, NSTA-SMC effectively suppresses sliding mode chattering and effectively solves the contradiction between the reaching speed of SMC and system chattering. Compared with STA-SMC, NSTA-SMC improves the system's response speed and antidisturbance ability without increasing the system chattering and further improving the system's dynamic quality.

From Figures 8 and 9, compared with PI, SMC1, SMC2, and STA-SMC, the control strategy proposed in this paper has the fastest torque response speed and smaller torque fluctuation. When dealing with external disturbance, it takes the least time to restore to the 


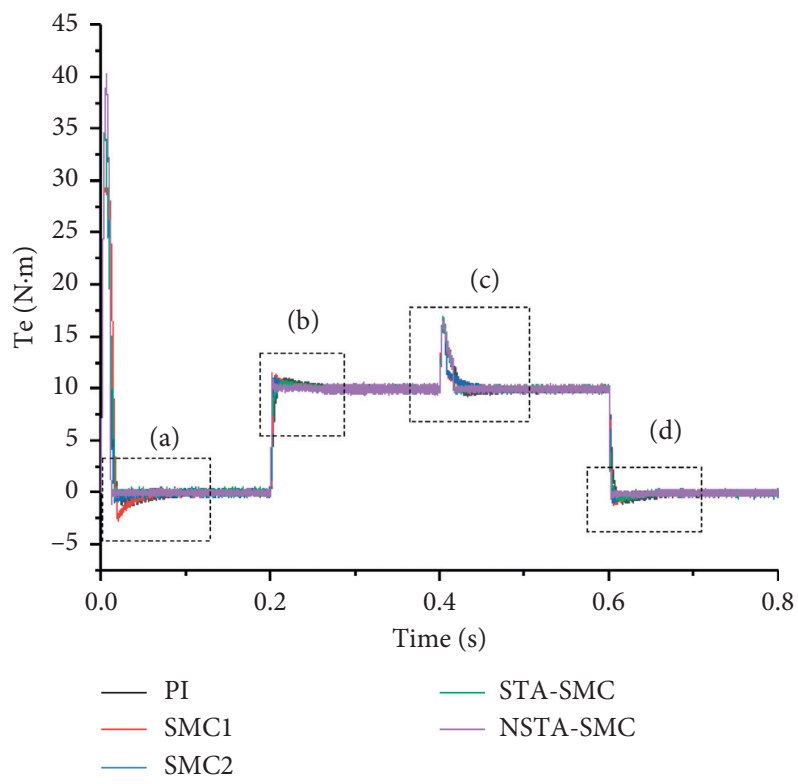

Figure 8: Torque response diagram.
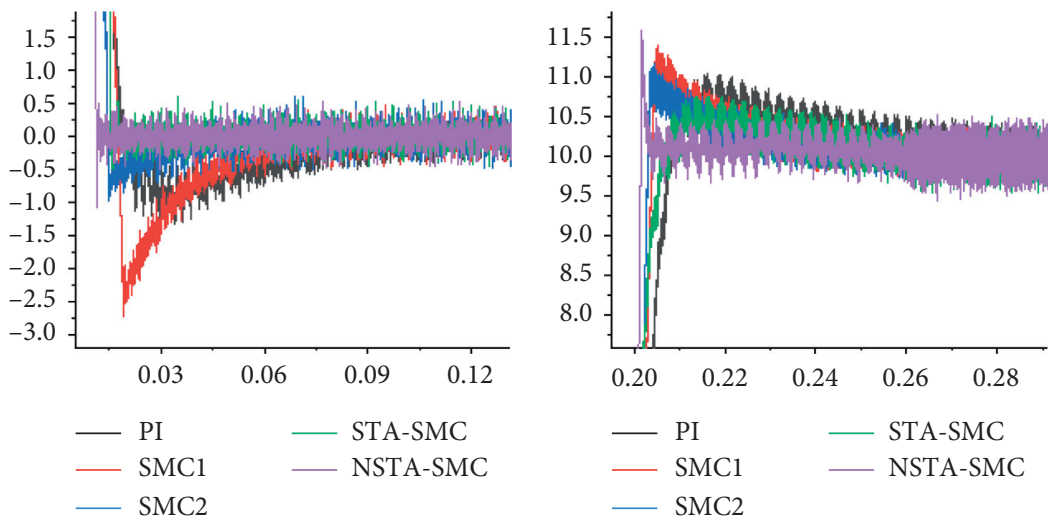

(a)

(b)
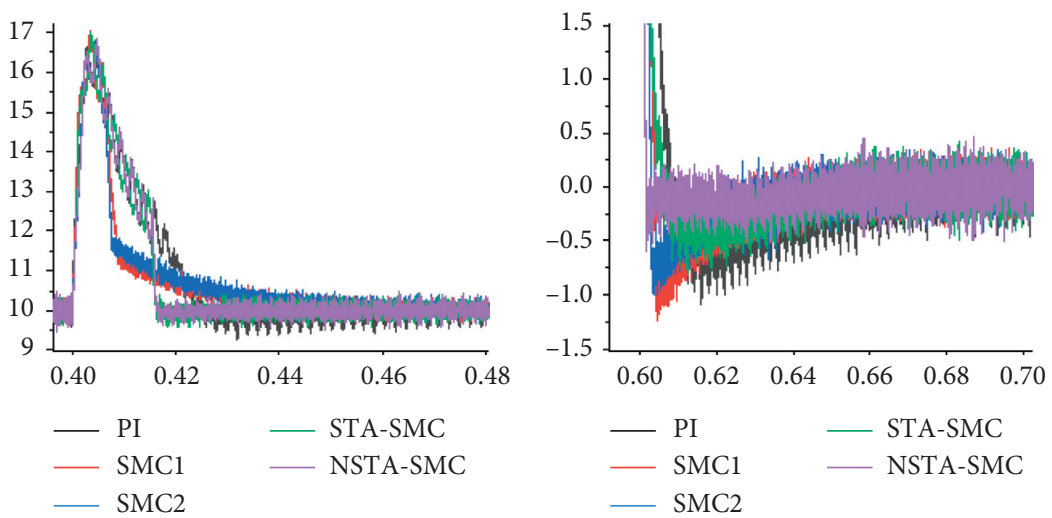

(c)

(d)

FIgURE 9: Enlargement of local torque response. 
original torque and has the best dynamic response performance.

\section{Conclusion}

(1) Compared with STA-SMC, NSTA-SMC with adaptive term effectively solves the problem of low reaching speed and poor antidisturbance ability caused by the square root calculation of proportional term in the original super twisting algorithm without increasing system chattering and improves the dynamic following performance of the system.

(2) Compared with the SMC based on the exponential reaching law, the NSTA-SMC effectively solves the contradictory problems between sliding mode chattering and reaching speed and antidisturbance ability under the premise of suppressing chattering and further improving the system control quality.

(3) Compared with PI, NSTA-SMC effectively improves the PI control excessive overshoot and poor antidisturbance ability and improves the dynamic and static quality of the system.

(4) At the same time, how to ensure the optimal sliding mode gain of the new super twisting algorithm has become the research focus to further improve the system performance.

\section{Data Availability}

The data used to was support the findings of this study are available from the corresponding author upon request.

\section{Conflicts of Interest}

The authors declare that there are no conflicts of interest related to the article.

\section{Acknowledgments}

This work was supported by Jilin Science and Technology Development Plan Project (20200403133SF).

\section{References}

[1] Y. Wang, Y. Feng, X. Zhang, J. Liang, and X. Cheng, "New reaching law control for permanent magnet synchronous motor with extended disturbance observer," IEEE Access, vol. 7, pp. 186296-186307, 2019.

[2] H. H. Choi, T. T. Vu, and J. W. Jung, "Digital implementation of an adaptive speed regulator for a PMSM," IEEE Transactions on Power Electronics, vol. 26, pp. 3-8, 2010.

[3] L. Yan, J. B. Son, and J. M. Lee, "PMSM speed controller using switching algorithm of PD and sliding mode control," in Proceedings of the 2009 ICCAS-SICE, Fukuoka, Japan, 2009.

[4] X. Zhang, L. Sun, and K. Zhao, "Sliding mode control of permanent magnet synchronous motor based on sliding mode observation of load torque," Proceedings of the CSEE, vol. 3, pp. 137-142, 2012.

[5] W. Gao, Theory and Design Method of Variable Structure Control, Science Press, Beijing, China, 1996.
[6] D. Lv and Z. Li, "Improved sliding mode observer control for permanent magnet synchronous motor," Electric Machines and Control Journal, vol. 1, pp. 1-10, 2021, in Chinese.

[7] Z. Li, "Integral sliding mode variable structure control of permanent magnet synchronous motor speed regulating system," Proceedings of the CSEC, vol. 34, pp. 431-437, 2014.

[8] I. C. Baik, K. H. Kim, and M. J. Youn, "Robust nonlinear speed control of PM synchronous motor using adaptive and sliding mode control techniques," IEE Proceedings-Electric Power Applications, vol. 145, pp. 369-376, 2002.

[9] Y. Feng, X. Yu, and F. Han, "High-order terminal slidingmode observer for parameter estimation of a permanentmagnet synchronous motor," IEEE Transactions on Industrial Electronics, vol. 60, no. 10, pp. 4272-4280, 2013.

[10] F. Xu, N. An, J. Mao, and S. Yang, "A new variable exponential power reaching law of complementary terminal sliding mode control," Complexity, vol. 2020, Article ID 8874813, 11 pages, 2020.

[11] D. Fu, X. Zhao, and H. Yuan, "High-precision motion control method for permanent magnet linear synchronous motor," IEICE Electronics Express, vol. 18, no. 9, 2021.

[12] X. Sun, L. Feng, K. Diao, and Z. Yang, "An improved direct instantaneous torque control based on adaptive terminal sliding mode for a segmented-rotor SRM," IEEE Transactions on Industrial Electronics, vol. 99, p. 1, 2020.

[13] X. Zhao and D. Fu, "Adaptive neural network nonsingular fast terminal sliding mode control for permanent magnet linear synchronous motor," IEEE Access, vol. 7, pp. 180361-180372, 2019.

[14] Y. Wang, F. Yong, and X. Yu, "High-order nonsingular terminal sliding mode control of uncertain multivariable systems," in Proceedings of the 2008 Conference of the IEEE Industrial Electronics Society, Taipei, Taiwan, 2008.

[15] Y. Feng, J. Zheng, X. Yu, and N. V. Truong, "Hybrid terminal sliding-mode observer design method for a permanentmagnet synchronous motor control system," IEEE Transactions on Industrial Electronics, vol. 56, no. 9, pp. 3424-3431, 2009.

[16] A. Levant, "Principles of 2-sliding mode design," Automatica, vol. 43, no. 4, pp. 576-586, 2007.

[17] Q. Hou, S. Ding, and X. Yu, "Composite super-twisting sliding mode control design for PMSM speed regulation problem based on a novel disturbance observer," IEEE Transactions on Energy Conversion, vol. 99, p. 1, 2020.

[18] Z. Li, S. Zhou, Y. Xiao, and L. Wang, "Sensorless vector control of permanent magnet synchronous linear motor based on self-adaptive super-twisting sliding mode controller," IEEE Access, vol. 7, pp. 44998-45011, 2019.

[19] Y. Chen, "Modified super-twisting algorithm with an antiwindup coefficient adopted in PMSM speed loop control," Energy Procedia, vol. 158, pp. 2637-2642, 2019.

[20] L. Zhang, S. Wang, and J. Bai, "Fast-super-twisting sliding mode speed loop control of permanent magnet synchronous motor based on SVM-DTC," IEICE Electronics Express, vol. 18, p. 1, 2020.

[21] P. Li and Z. Zheng, "Analysis of convergence of super-twisting algorithm based on quasi-quadratic Lyapunov function," Control and Decision, vol. 26, pp. 949-952, 2011.

[22] V. K. Tripathi, A. K. Kamath, L. Behera, N. K. Verma, and S. Nahavandi, "Finite-time super twisting sliding mode controller based on higher-order sliding mode observer for real-time trajectory tracking of a quadrotor," IET Control Theory \& Applications, vol. 14, no. 16, pp. 2359-2371, 2020.

[23] L. Yu, Robust Control-Linear Matrix Inequality, Tsinghua University Press, Beijing, China, 2002. 